\title{
Anterior surgical approaches to the thoracic outlet
}

\author{
M. Blair Marshall, MD, ${ }^{a}$ John C. Kucharczuk, MD, ${ }^{b}$ Joseph B. Shrager, MD, and Larry R. Kaiser, $M D^{b}$
}

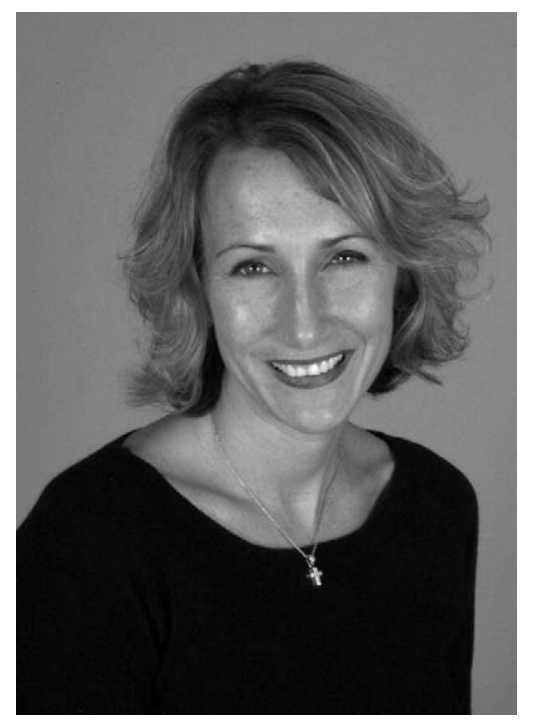

Dr Marshall

Objectives: The anatomy of the thoracic outlet is complex, and the optimum surgical approach to pathologic disease at this location is controversial. Although the Dartevelle approach to the apex seems to be a safer and more direct approach, this technique has not been widely adopted in the United States. We have used this approach for pathologic disease at the thoracic outlet and modified it. Our experience is described in this article.

Methods: A retrospective review was performed on all patients who underwent an anterior approach between December 1997 and May 2003.

Results: There were 42 patients who underwent anterior approaches to pathologic disease at the level of the outlet. Diagnosis included apical non-small cell lung cancers (20 patients), osteosarcoma (2 patients), spinal cord compression (5 patients), solitary metastasis (4 patients), and benign lesions (11 patients). There were 22 female and 20 male patients with ages ranging from 26 to 82 years (mean age, 54.6 years). There were 25 complications in 14 patients and 1 in-hospital death. A transmanubrial approach was used in 14 patients, the standard Dartevelle technique was used in 8 patients, and a transclavicular approach with reapproximation of the clavicle was used in 20 patients. Reapproximation failed in 5 patients ( $3 / 3$ patients who underwent fixation with mini-plates and 2/17 patients with sternal wires).

Conclusions: The anterior approach is a useful adjunct to a thoracic surgeon's armamentarium. When a transclavicular approach is optimal, division and reapproximation of the clavicle are feasible. In our experience, reapproximation with wires is superior to plates and screws.

From the ${ }^{a}$ Division of Thoracic Surgery, Department of Surgery, Georgetown University Medical Center, Washington, DC; ${ }^{\text {b}}$ Section General Thoracic Surgery, Department of Surgery, University of Pennsylvania School of Medicine, Philadelphia, Penn.

Received for publication Oct 6, 2005; accepted for publication Jan 24, 2006.

Address for reprints: M. Blair Marshall, MD, Chief, Division of Thoracic Surgery, Department of Surgery, Georgetown University Hospital, 3800 Reservoir Road (E-mail: mbm5@gunet.georgetown.edu).

J Thorac Cardiovasc Surg 2006;131:1255-60 $0022-5223 / \$ 32.00$

Copyright (C) 2006 by The American Association for Thoracic Surgery

doi:10.1016/j.jtcvs.2006.01.044

$\mathrm{S}$ urgical pathologic disease at the level of the thoracic outlet represents a technical challenge for most thoracic surgeons, in part, because of the complex bony anatomy protecting this anatomic region. Pathologic disease requiring surgical access to this region is varied and includes both malignant and benign disease.

Since the classic article by Shaw and colleagues in the $1960 \mathrm{~s},{ }^{1}$ the standard approach to superior sulcus tumors has been a posterolateral one. For many, there are clear advantages to this approach. Because pathologic disease in this location is rare, most surgeons are comfortable with an approach that is an extension of one they perform every day. In addition, the entire procedure can be performed through a single incision. However, the posterior approach does not allow for optimal exposure or control of the neurovascular structures with complete oncologic clearance. To obtain exposure of the outlet structures and first rib requires division of several posterior chest wall muscles; these must be divided before determining whether the disease is entirely resectable.

For superior sulcus lesions, the transclavicular approach described by Dartevelle and colleagues ${ }^{2}$ has distinct advantages. This approach is performed through an anterior L-shaped cervicotomy incision. The sternocleidomastoid is dissected away from the clavicle and reflected as a myocutaneous flap. The inferior belly of the omohyoid is divided, and the scalene fat pad is evaluated. The superior mediastinum 
is directly palpated to assess the extent of disease. Once the disease is determined, the medial half of the clavicle is divided.

Because these tumors may involve the neurovascular structures and complicate the resection, this approach provides optimal exposure of both the proximal and distal neurovascular structures. The scalene fat pad and lymph nodes can readily be dissected and evaluated initially without the morbidity of a large posterolateral incision. There is little morbidity associated with an anterior exposure, even if the tumor is deemed unresectable.

Many surgeons have not readily adopted this technique, initially in part because of an additional thoracotomy required for the parenchymal resection. However, subsequent publications have demonstrated that anatomic pulmonary resection can be adequately and safely performed through the anterior chest wall defect created at the time of the resection. ${ }^{3}$

Inherent to this approach is the resection of the medial half of the clavicle. Fracture of the clavicle has recently been associated with significant long-term sequelae in up to $42 \%$ of patients. ${ }^{4}$ The impact of the Dartevelle transclavicular approach on long-term upper extremity function has not been formally evaluated. For this approach, the scalenes are divided and the apical chest wall is resected, as is part of the procedure. One would expect preservation of the sternoclavicular joint to be important for shoulder stabilization and arm function in these patients. Loss of the anterior clavicular strut in addition to other supporting structures of the shoulder girdle can allow for collapse of the glenohumeral joint. Because of this disadvantage, some authors have described modifications to the standard Dartevelle approach in an attempt to preserve the clavicle and its function; however, the experience is limited. ${ }^{5-7}$

As use of the anterior approach has become more widespread in Europe, surgeons have applied this approach to other areas of pathologic disease at the level of the thoracic outlet. ${ }^{5,8-10}$ Several modifications have been made to suit the clinical situation. Although the skin incision may be similar for these, the subsequent dissection is dependent on the planned objectives of the procedure.

When approaching pathologic disease at the thoracic outlet, we have found the anterior approach to provide optimal exposure overall. We have also found it necessary to modify the approach in accordance with the pathologic disease and planned procedure. We were impressed by the excellent exposure provided with this technique but were concerned with removing the medial half of the clavicle, a supportive structure without pathologic involvement. This was particularly true for patients with apical parenchymal lesions involving the chest wall. In this group of patients, it seemed paramount to preserve as much shoulder support as possible. We have modified the technique to maintain clav-
TABLE 1. Resections for cancer

No. of patients

NSCLC

Stage IIB

Stage IIIA 3

Stage IIIB

Stage IV* (solitary brain metastasis)

Local recurrence

Other

Primary osteosarcoma first rib

Local recurrence, extraosseous osteosarcoma

Other solitary metastasis

NSCLC, Non-small cell lung cancer.

icle function. For patients who do not require chest wall resection, it is also not necessary to divide the clavicle; thus, the sternoclavicular joint may be left intact. During the past few years, we have incorporated the anterior approach for most pathologic disease at the thoracic outlet and report our experience with and modifications of this technique in this article.

\section{Methods}

A retrospective review was performed on all patients who underwent an anterior approach at our institution between December 1999 and May 2003. Expedited approval from our institutional review board was obtained. Anterior approaches to the apex included those patients who underwent a transmanubrial approach, standard transclavicular approach (Dartevelle), or transclavicular approach with reapproximation of the clavicle.

\section{Patients and Results}

There were 42 patients who underwent anterior approaches. The patients were categorized into 3 separate groups based on pathologic disease: benign lesions (11), spine exposure (5), and cancer (26): primary tumors (18), solitary metastasis (4) and local recurrences (4) (Table 1).

Primary anterior approaches used were transmanubrial in 14 patients, transclavicular with removal of the medial third of the clavicle in 8 patients, and transclavicular with reapproximation of the clavicle in 20 patients. Clavicles were reapproximated with wire in 17 patients and mini-plates and screws in 3 patients.

For patients with non-small cell lung cancers, neoadjuvant therapy was used in 10, preoperative chemotherapy and radiation therapy in 5, radiation alone in 4, and chemotherapy alone in 1 . Additional procedures performed included mediastinoscopy in 12 patients. The apical chest wall was resected in all patients with non-small cell lung cancer, both primary and local recurrences: ribs 1 to 3 in 16 patients and ribs 1 to 2 in 4 patients. When necessary, chest wall reconstruction was performed with methylmethacrylate and Marlex mesh or Marlex mesh alone. Patients with a primary non-small cell lung cancer (17) underwent pulmonary resection with the primary approach. There were 15 upper lobectomies, 1 lingular sparring left upper lobectomy, and 1 wedge resection performed for primary non-small cell lung cancers. For 
TABLE 2. Diagnoses: Benign lesions

\begin{tabular}{lc}
\hline Pathology & No. of patients \\
\hline Schwannoma & 4 \\
Parathyroid adenoma & 2 \\
Inflammatory pseudotumor & 2 \\
Fibrous dysplasia (first rib) & 1 \\
Neurofibroma & 1 \\
Desmoid & 1 \\
\hline
\end{tabular}

patients with recurrent local disease (3), 1 wedge resection and 2 superior segmentectomies were performed. Two of these patients had their parenchymal resection performed through the anterior approach, and 1 patient required a repeat muscle-sparing thoracotomy for superior segmentectomy after adhesiolysis.

Additional procedures in patients with apical non-small cell lung cancers included subclavian vein resection in 4 , resection of the subclavian artery with polytetrafluoroethylene (Gore-Tex; WL Gore \& Associates, Flagstaff, Ariz) reconstruction in 1, vertebral body resection and reconstruction in 3 , and resection of the $\mathrm{T} 1$ nerve root in 4 . The stellate ganglion was resected in 2 patients, both with preoperative Horner's syndrome. One patient underwent resection and reconstruction of the superior vena cava. Pathologic staging of the patients with primary non-small cell lung cancer is detailed in Table 2 . The subclavian vein was resected in 2 patients (1 patient with a fourth recurrence of a desmoid tumor and 1 patient with an inflammatory pseudotumor and venous thrombosis).

Twenty-five complications occurred in 14 patients. Clavicle dehiscence occurred in 5 of 20 patients who underwent clavicle reapproximation. Fusion failed in all 3 patients who underwent mini-plate fixation, and this technique was abandoned. Wire fixation also failed in 2 patients, 1 who refused to wear an arm sling. One patient underwent resection of the clavicle, and 1 patient underwent repeat wire fixation.

Pneumonia developed in 4 patients; 3 of these were patients with end-stage metastatic disease in whom exposure was provided for neurosurgical procedures. Five patients experienced atrial arrhythmias that were controlled with medical therapy. One patient required a blood transfusion because of postoperative bleeding. A prolonged air leak developed in 3 patients, 2 of whom underwent repeat resection for local recurrence after failing $5600 \mathrm{cG}$. In 1 patient, the pectoralis major was harvested through the cervicothoracic incision and placed in the chest. The second patient underwent a cervicothoracic approach followed by axillary thoracotomy for superior segmentectomy. To treat the prolonged air leak, the latissimus dorsi was harvested through the axillary incision and placed in the chest as previously described. ${ }^{11}$ Both of these leaks resolved, and the patients were discharged without tubes.

Chronic pain and upper extremity weakness occurred in 3 patients who underwent the standard Dartevelle approach. It is unclear whether this was related to the extent of surgical resection or the approach. Wound infection associated with clavicle disruption occurred in 2 patients. Both of these patients underwent reoperation for removal of the medial head of the clavicle; neither required long-term antibiotics. A suspected cerebrospinal fluid leak occurred in 1 patient, who responded to conservative therapy. There was 1 death. This patient had metastatic small-cell carci- noma and underwent exposure for upper thoracic spine stabilization. Urosepsis developed in the patient, followed by pneumonia; the patient had a "do not resuscitate" status and was allowed to die.

\section{Technique}

The approach was anterior in all patients. Depending on the intent, the operative procedure was chosen to suit each clinical case. For those patients requiring intrathoracic exposure, either for spine exposure or pathologic disease not involving the chest wall, the sternoclavicular joint was spared and a transmanubrial approach was used. In these patients, an L-shaped skin incision was used. The incision coursed along the anterior border of the sternocleidomastoid to the sternal notch, and out below the clavicle toward the deltopectoral groove. The manubrium was divided in the midline and brought out to either a first or second interspace thoracotomy. The level of thoracotomy was dictated by the level of exposure required.

For those patients with pathologic disease requiring apical chest wall resection, a transclavicular approach as described by Dartevelle and colleagues ${ }^{2}$ was used initially. As our experience grew, the transclavicular technique with clavicular reapproximation was used. Two types of clavicular fixation were attempted. The mini-plate techniques failed in 3 of 3 patients and was abandoned. Wire fixation is described next.

\section{Transclavicular Technique with Reapproximation}

The patient is positioned supine, the head is turned away from the operative side, and the patient is prepped from ear to below the costal margin. The standard L-shaped incision is made (Figure 1). The strap and sternocleidomastoid muscles are dissected away from of the head of the clavicle, and it is dissected circumferentially. A $4 \times 8$ sponge is drawn though the space between the clavicle and first rib. Next, an oscillating saw is used to make an oblique osteotomy through the largest area of the clavicular head (Figure 2). The clavicle is lifted with a Rultract retractor (Rultract Inc., Independence, Ohio), and the dissection proceeds as for the standard transclavicular approach.

The scalene fat pad is dissected, and the great vessels and brachial plexus are palpated to determine the extent of disease. The jugular vein is dissected first. The dissection is continued down toward the subclavian vein and its branches. The thoracic duct is ligated on the left side when appropriate. The proximal and distal vessels beyond the tumor involvement are dissected for control. One should ensure catheter access away from the disease when anticipating vascular involvement. We usually place our vascular access in the femoral vessels or contralateral upper extremity.

The anterior rib attachments are divided first. The thoracotomy is made along the intercostal at the lowest rib being resected. This provides intrathoracic exposure and the division of the remaining posterior rib attachments. With this exposure, the posterior first rib attachments are easily divided. The remainder of the posterior chest wall attachments are approached from both the superior and intrathoracic exposures. As the segment of chest wall is completely detached, it is dropped into the defect created and the parenchymal and lymph node dissections are performed through this exposure. Once the resection is complete, the clavicle is reapproximated with 2 number 5 stainless-steel wires. The wound is closed in layers, and the patient's arm is placed in a sling for 6 weeks. 


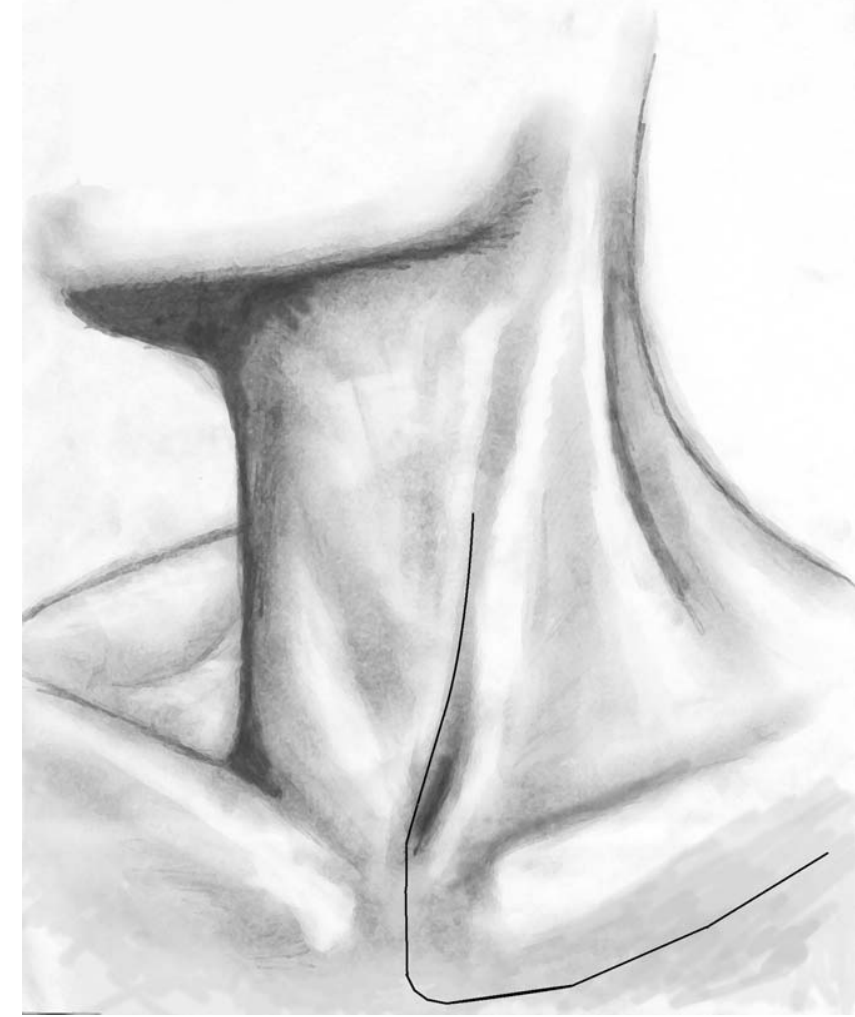

Figure 1. L-shaped skin incision for the anterior approach. The superior aspect courses along the anterior border of the sternocleidomastoid to the manubrium, then curves out laterally below the clavicle. For approaches that involve resection of the chest wall, the infraclavicular aspect of the incision should be adjusted inferiorly.

\section{Discussion}

Because of the natural anterior curve of the upper thoracic and lower cervical spine, the apex of the chest and thoracic outlet is relatively anterior. For the apical ribs, the posterior aspect of these ribs are more cephalad than their anterior counterparts. These are important anatomic relationships. When working through the posterolateral approach, one must divide several posterior chest wall muscles to elevate the scapula to then come up and over the posterior first rib to expose the outlet, scalene fat pad, and neurovascular structures. As demonstrated in Figure 3, A, the outlet structures lie just posterior to the clavicle.

The Dartevelle transclavicular approach allows for evaluation of the scalene fat pad, brachial plexus, and subclavian vessels early on in the dissection. There is little morbidity associated with this incision and approach, making it an attractive option for surgical exposure. In addition, if the disease is deemed unresectable, there is little associated morbidity for the patient. During the past few years, we have been impressed with the ease of exposure of the

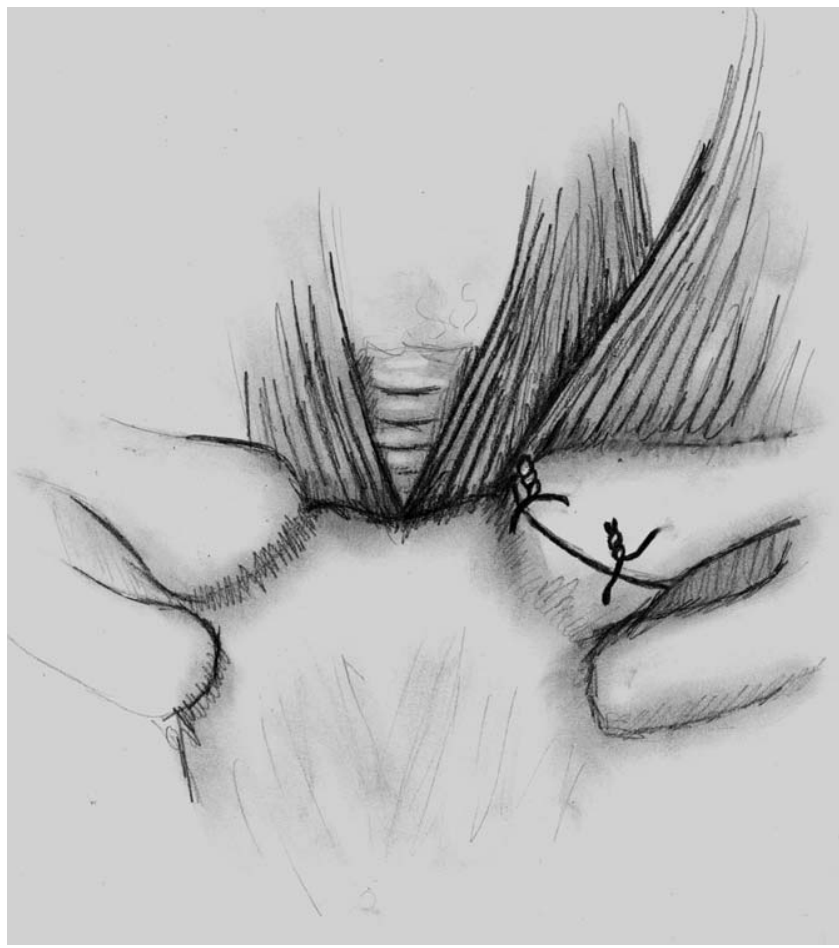

Figure 2. Location of osteotomy, dividing the head of the clavicle through the area of largest diameter with reapproximation with wires.

thoracic outlet and complex pathologic disease through the cervicothoracic incision. We continue to use this as the exposure of choice for pathologic disease at the level of the outlet.

In using an anterior approach, we established an algorithm to aid in the operative planning for this approach. The potential resection of the chest wall is the key point to define when planning the approach. If chest wall resection is necessary, as in a Pancoast lesion, then the defect created by taking down the chest wall is used for the intrathoracic exposure and formal pulmonary dissection. Thus, the clavicle will have to be divided to begin the dissection. For procedures requiring chest wall resection, we carefully review the preoperative computed tomography to determine which ribs will be resected. This helps to plan the skin incision. For first and second rib resections, the planned skin incision courses along the inferior aspect of the clavicle. If the anterior aspect of the third rib requires resection, then the skin incision should be adjusted inferiorly as appropriate. If this is not done, the soft tissues of the anterior chest wall, no longer supported by the ribs, tend to obstruct the exposure for the parenchymal resection. Although one might think that this approach is not suitable for apical tumors involving the posterior aspect of the chest wall, we have not found this to be the case. As demonstrated in 


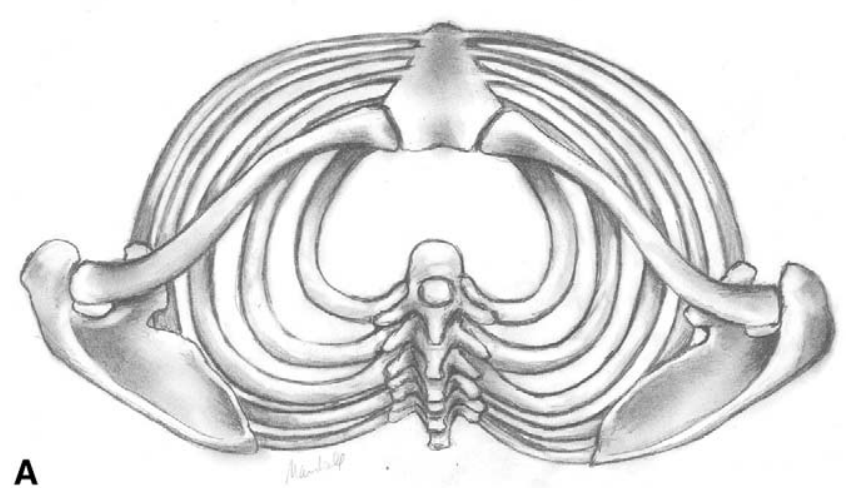

A

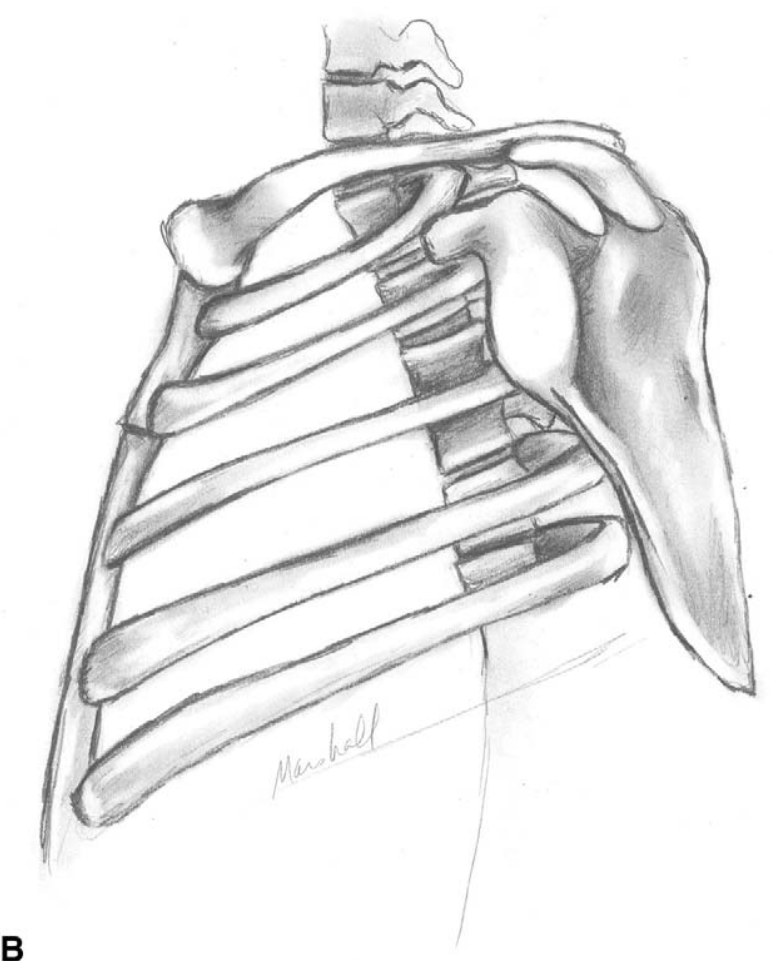

Figure 3. A, Thoracic cage viewed from above, demonstrating the location of the thoracic outlet, just posterior to the clavicle, and fairly anterior in location. B, Anterior curve of the lower cervical and upper thoracic spine. This curve, along with the cephalad course of the posterior apical ribs, allows even posterior apical tumors to be resected through this approach.

Figure 3, $B$, for the superior ribs, the posterior rib heads are more cephalad than their corresponding anterior components. In particular, the posterior aspect of the first rib, in relation to the rest of the chest wall, is anterior (Figure 4). Thus, by dividing the first rib alone, taking the intercostals and other chest wall attachments, excellent exposure of the posterior ribs 2 and 3 is provided.

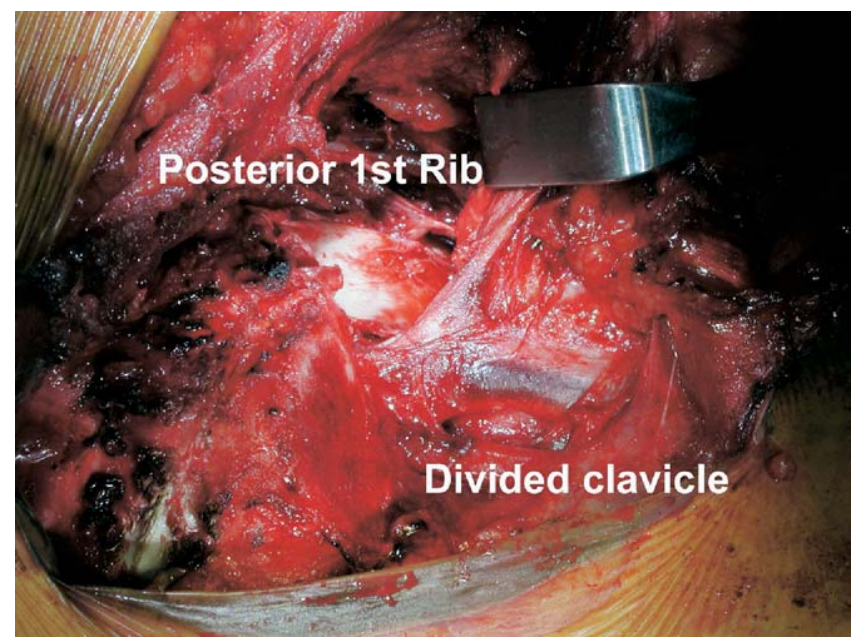

Figure 4. Intraoperative photograph demonstrates the close proximity of the posterior first rib to the head of the clavicle (divided).

One of the disadvantages to the classic anterior approach described by Dartevelle and colleagues ${ }^{2}$ is the sacrifice of the clavicle, a supportive structure without pathologic involvement. We believe that this support for the glenohumeral joint is important in patients undergoing resection of most of the apical chest wall. We have modified the transclavicular technique to optimize reapproximation. For the rewiring technique, an oblique osteotomy through the largest part of the head of the clavicle provides maximal bone apposition. Other modifications to the technique include the use of an oscillating saw to divide the clavicle. As opposed to the Gigli saw, the oscillating saw provides for more control of the osteotomy, creates a smoother cut surface, and does not destroy as much bone with its use. When mini-plates were used for reconstruction the clavicle was divided along the shaft, one of the narrowest regions of the clavicle. This, combined with the type of plates used, may have contributed to the high failure rate observed with this technique. Others have used plate fixation for reconstruction of the transected midshaft of the clavicle with success. ${ }^{6}$

If the chest wall will not be resected as part of the management of the pathologic disease, then one does not have this defect through which to work in lieu of a thoracotomy. For intrathoracic pathologic diseases, including vertebral disease, one must create the intrathoracic exposure through an anterior thoracotomy. This is done with a modified thoracosternotomy, a trasnmanubrial approach brought out through either the first or second intercostal space depending on the location of the pathologic disease and planned procedure. Thus, one can avoid division of the clavicle and the associated morbidity. 
Currently described anterior approaches to the thoracic inlet include the Dartevelle technique and several modifications of it. Most of the literature surrounding this technique, other than Dartevelle and colleagues' series $^{2}$ and Goldstraw's experience, includes case reports with description of a new technique and other limited series. ${ }^{5,7,10}$ In this article, we highlighted the complex anatomic relationships favoring the anterior approach to the thoracic outlet. In addition, we described our experience with this approach using both the transclavicular and transmanubrial techniques. We emphasized the key points to define when addressing thoracic outlet pathologic disease through an anterior cervicothoracic approach. We believe this approach to be superior to other approaches for the management of superior sulcus tumors and additional pathologic disease at the level of the thoracic outlet, and we encourage others to adopt these techniques.

\section{References}

1. Shaw RR, Paulson DL, Kee JL. Treatment of superior sulcus tumor by irradiation followed by resection. Ann Surg. 1961;154:29-40.
2. Dartevelle PG, Chapelier AR, Macchiarini P, et al. Anterior transcervical thoracic approach for radical resection of lung tumors invading the thoracic inlet. J Thorac Cardiovasc Surg. 1993;106:1025-34.

3. Macchiarini P, Chapelier AR, Monnet I, et al. Extended operations after induction therapy for stage IIIb (T4) non-small cell lung cancer. Ann Thorac Surg. 1994;57:966-73.

4. Ledger M, Leeks N, Ackland T, Wang A. Short malunions of the clavicle: an anatomic and functional study. J Shoulder Elbow Surg. 2005; 14:349-54.

5. Grunenwald D, Spaggiari L. Transmanubrial osteomuscular sparing approach for apical chest tumors. Ann Thorac Surg. 1997;63:563-6.

6. Nazari S. Transcervical approach (Dartevelle technique) for resection of lung tumors invading the thoracic inlet, sparing the clavicle. $J$ Thorac Cardiovasc Surg. 1996;112:558-60.

7. Papsin BC, Freeman JL, Davis A, Bell RS. Transclavicular approach to the neck, thoracic inlet and axilla. Arch Otolaryngol Head Neck Surg. 1995;121:984-7.

8. Rusca M, Carbognani P, Bobbio P. The modified "hemi-clamshell" approach for tumors of the cervicothoracic junction. Ann Thorac Surg. 2000;69:1961-3.

9. Ladas G, Rhys-Evans PH, Goldstraw P. Anterior cervical-trans sternal approach for resection of benign tumors of the thoracic inlet. Ann Thorac Surg. 1999;67:785-9.

10. Vaqnakesa T, Goldstraw P. Antero-superior approaches in the practice of thoracic surgery. Euro J Cardiothorac Surg. 1999;15:774-80.

11. Marshall MB, Kaiser LR, Kucharczuk. Simple technique for maximal thoracic muscle harvest. Ann Thorac Surg. 2004;77:1465-6. 